\title{
Modeling the Drainage of aVertical Thin Liquid Film
}

\author{
Joseph G. Abdulahad \\ College of Education \\ University of Dohuk
}

Received on:13/9/2009

Accepted on:11/4/2010

\begin{abstract}
In this paper, we consider the drainage of a vertical thin liquid film and we study the case of instability in gravity driven flow of a vertical thin films. Throughout this work, we assumed that the fluid thickness is constant far behind the front and we neglect the thickness of the film at the beginning of the motion. The equation of the film thickness is obtained analytically, using the similarity method by which we can isolate the explicit time dependence and then the shape of the film will depend on one variable only.

Keywords: Governing Equations, Similarity method.

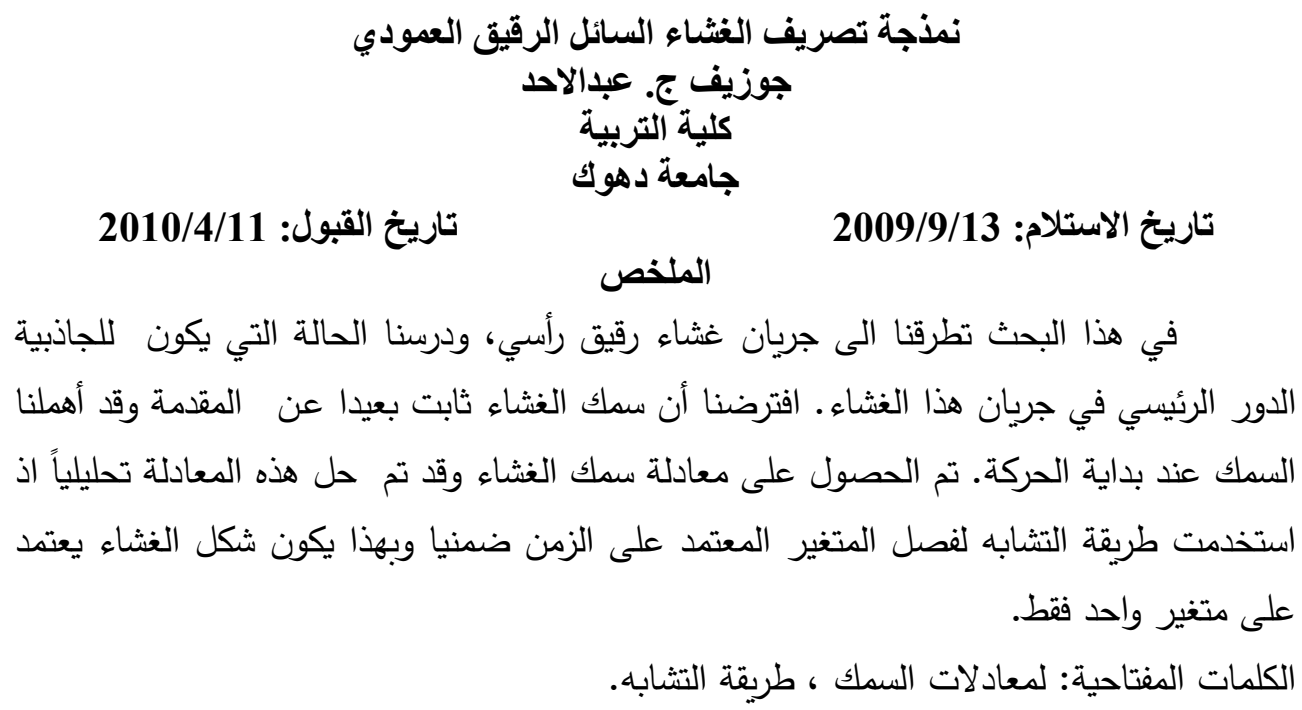

\section{Introduction:}

We expressed here some of the theoretical aspects of the instability development in a vertical thin liquid films in two dimensional coordinate system. There are different types of phenomena that can occur, such as drainage, details of rupture, non-Newtonian surface properties [2] and 
moving contract lines in thin films [1]. These phenomena can help to describe the physical processes that occur in our real world.

Kondic and Diez [7] have studied the case of contact line instability of thin liquid films on an inclined plane. [3] have studied the global models for moving contact lines. Huppert [4] considered the flow and instability of a viscous current down a slope. It is obvious that gravity has a significant effect on the flow of this films but also here we present some other forces that also have an important effect on the flow such as (viscous, surface tension) and for different types of fluids. Different fluid-solid combinations have different wetting properties that influence the shape of the patterns [5]. The flow of thin films is relevant to a number of different fields such as engineering, biology and chemicals [6]. These flows can be driven by gravitational forces [8], studied the contact line instability of thin films flows with constant flux configuration, which presents theoretical, computational and experimental aspects of the instability development in such flows.

\section{Governing Equations:}

Let $q=q(u, w)$ denotes the fluid velocity, where $u$ and $w$ are the velocity components in $\mathrm{x}$ and $\mathrm{z}$ directions respectively. Let $z=h(x, t)$ be the equation of the vertical thin liquid films as shown in Figure (1) and the flow is in $\mathrm{x}$ direction.

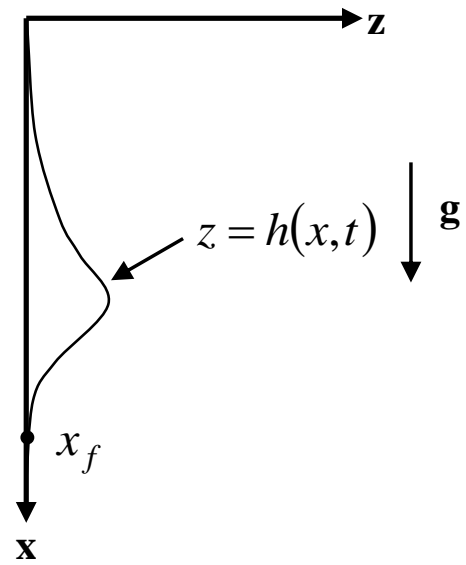

Figure (1): Sketch of the flow in two-dimensional geometry, where the capillary ridge is just behind the flow front

The continuity equation is given by:

$\frac{\partial u}{\partial x}+\frac{\partial w}{\partial z}=0$ 
The Navier-Stokes equations in $x$ and $y$ directions respectively for a vertical thin liquid film is give by:

$$
\begin{aligned}
& \rho\left(\frac{\partial u}{\partial t}+u \frac{\partial u}{\partial x}+w \frac{\partial u}{\partial z}\right)=-\frac{\partial P}{\partial x}+\mu\left(\frac{\partial^{2} u}{\partial x^{2}}+\frac{\partial^{2} u}{\partial z^{2}}\right)+\rho g \\
& \text { And } \\
& \rho\left(\frac{\partial w}{\partial t}+u \frac{\partial w}{\partial x}+w \frac{\partial w}{\partial z}\right)=-\frac{\partial P}{\partial z}+\mu\left(\frac{\partial^{2} w}{\partial x^{2}}+\frac{\partial^{2} w}{\partial z^{2}}\right)
\end{aligned}
$$

Where $\rho, \mu$ are the density, viscosity of fluid and $P$ the pressure.

Since the Reynolds member is the very small and so the inertia terms in the Navier-Stokes equation can be neglected and thus equations (2) and (3) become

$$
\begin{aligned}
& \frac{\partial P}{\partial x}=\mu\left(\frac{\partial^{2} u}{\partial x^{2}}+\frac{\partial^{2} v}{\partial z^{2}}\right)+\rho g \\
& \frac{\partial P}{\partial z}=\mu\left(\frac{\partial^{2} w}{\partial x^{2}}+\frac{\partial^{2} w}{\partial z^{2}}\right)
\end{aligned}
$$

The boundary conditions to be imposed are as follows:

The no-slip condition gives

$$
\text { at } z=0, u=0
$$

The shear stress condition on the surface vanishes, that is:

at $z=h, \frac{\partial u}{\partial z}=0$

Also the pressure form the Laplace-Young condition states that at $z=h$, we have

$$
P=-\sigma k+P \text { 。 }
$$

Where $k$ is the curvature of the surface of the film, $\sigma$ is the surface tension and $P_{\circ}$ is the atmospheric pressure in the air phase.

We introduce the following non-dimensional variables as follow:

$$
\begin{gathered}
x=L \bar{x}, \quad z=H \bar{z}, u=U \bar{u}, w=\in U \bar{w}, P=\frac{\mu U}{L} \bar{P}, \in=\frac{H}{L}<<1, \\
h=H \bar{h} .
\end{gathered}
$$

We can neglect the term $\frac{\partial^{2} u}{\partial x^{2}}$, since

$\frac{\partial^{2} u}{\partial x^{2}}=\frac{1}{L^{2}} \frac{\partial^{2} u}{\partial x^{2}} \leq \frac{\partial^{2} u}{\partial z^{2}}=\frac{1}{H^{2}} \frac{\partial^{2} u}{\partial z^{2}}$ 
and so equations (4) and (5) become respectively

$\frac{\partial P}{\partial x}=\mu \frac{\partial^{2} u}{\partial z^{2}}+\rho g$

and

$\frac{\partial P}{\partial z}=0$

Equation (10) ensures that the pressure is a function of $x$ and $t$ only.

Now by using the non-dimensional variables, equations (1) and (9) in nondimensional form becomes.

$\frac{\partial \bar{u}}{\partial \bar{x}}+\frac{\partial \bar{w}}{\partial \bar{z}}=0$

and

$\frac{\partial \bar{P}}{\partial \bar{x}}=\frac{1}{\epsilon^{2}} \frac{\partial^{2} \bar{u}}{\partial \bar{z}^{2}}+\frac{\rho g L^{2}}{\mu U}$

The boundary conditions (6), (7) and (8) in non dimensional form become

$$
\text { At } \begin{aligned}
\bar{z} & =0, \quad \bar{u}=0 \\
\bar{z} & =\bar{h}, \quad \frac{\partial \bar{u}}{\partial \bar{z}}=0 \\
\bar{P} & =\frac{\varepsilon}{C a} \frac{\partial \bar{h}^{2}}{\partial \bar{x}^{2}}+P_{0}
\end{aligned}
$$

Where the Bond number $\beta$ and the capillary number $(\mathrm{Ca})$ are defined as:

$$
\beta=\frac{\rho g L^{2}}{\sigma}, \text { and } \quad C a=\frac{\mu U}{\sigma}
$$

Equation (9) in non-dimensional form gives

$\frac{\partial \bar{P}}{\partial \bar{x}}=\frac{1}{\epsilon^{2}} \frac{\partial^{2} \bar{u}}{\partial \bar{z}^{2}}+\frac{\beta}{C a}$

Integrating equation (16) with respect to $\bar{z}$, we get

$\frac{\partial \bar{P}}{\partial \bar{x}} \bar{z}=\frac{1}{\epsilon^{2}} \frac{\partial \bar{u}}{\partial \bar{z}}+\frac{\beta}{C a} \bar{z}+k_{1}(\bar{x}, t)$

Form the boundary condition (14), we have

$\frac{\partial \bar{P}}{\partial \bar{x}} \bar{h}=\frac{\beta}{C a} \bar{h}+k_{1}(\bar{x}, t)$

or 
$k_{1}(\bar{x}, t)=\frac{\partial \bar{P}}{\partial \bar{x}} \bar{h}-\frac{\beta}{C a} \bar{h}$

substituting (18) into (17), we get

$\frac{\partial \bar{P}}{\partial \bar{x}}(\bar{z}-\bar{h})=\frac{1}{\in^{2}} \frac{\partial \bar{u}}{\partial \bar{z}}+\frac{\beta}{C a}(\bar{z}-\bar{h})$

integrating equation (19) with respect to $\bar{z}$ we have

$\frac{1}{2} \frac{\partial \bar{P}}{\partial \bar{x}}(\bar{z}-\bar{h})^{2}=\frac{1}{\epsilon^{2}} \bar{u}+\frac{\beta}{2 C a}(\bar{z}-\bar{h})^{2}+k_{2}(\bar{x}, t)$

Using the boundary condition (13), and from equation (20), we have

$k_{2}(\bar{x}, t)=\frac{1}{2} \bar{h}^{2}\left(\frac{\partial \bar{P}}{\partial \bar{x}}-\frac{\beta}{C a}\right)$

Equations (20) and (21) and after some simplifications, give

$\bar{u}=\epsilon^{2}\left(\frac{\partial \bar{P}}{\partial \bar{x}}-\frac{\beta}{C a}\right)\left(\frac{\bar{z}^{2}}{2}-\bar{h} \bar{z}\right)$

The average velocity over the film thickness is given by

$\bar{u}_{1}=\frac{1}{\bar{h}} \int_{0}^{\bar{h}} \bar{u} d \bar{z}$

Equations (22) and (23), give

$\bar{u}_{1}=\frac{\epsilon^{2}}{\bar{h}}\left(\frac{\partial \bar{P}}{\partial \bar{x}}-\frac{\beta}{C a}\right)\left[\frac{\bar{z}^{3}}{6}-\frac{1}{2} \bar{h}^{2}\right]_{0}^{\bar{h}}$

or

$\bar{u}_{1}=\frac{\epsilon^{2} \bar{h}^{2}}{3}\left(\frac{\partial \bar{P}}{\partial \bar{x}}-\frac{\beta}{C a}\right)$

From the conservation of mass and since the free surface is a stream line, then the derivative following the motion $\frac{D \bar{h}}{D t}$ must be vanishes, thus

$\frac{D \bar{h}}{D t}=\frac{\partial \bar{h}}{\partial t}+\bar{u}_{1} \frac{\partial \bar{h}}{\partial \bar{x}}+\bar{w} \frac{\partial \bar{h}}{\partial \bar{z}}=0$

Since $\bar{h}$ is a function of $\bar{x}$ and $t$ only, equation (25), then become

$\frac{\partial \bar{h}}{\partial t}+\bar{u} \frac{\partial \bar{h}}{\partial \bar{x}}=0$

Now equations (24) and (26), give

$\frac{\partial \bar{h}}{\partial t}-\frac{\epsilon^{2} \bar{h}^{2}}{3}\left(\frac{\partial \bar{P}}{\partial \bar{x}}-\frac{\beta}{C a}\right) \frac{\partial \bar{h}}{\partial \bar{x}}=0$ 
The curvature of the surface is given by

$k=\frac{\partial^{2} \bar{h}}{\partial \bar{x}^{2}}\left[1+\left(\frac{\partial \bar{h}}{\partial \bar{x}}\right)^{2}\right]^{-3 / 2}$

Since for thin liquid films of small slope, that is since $\frac{\partial \bar{h}}{\partial \bar{x}}$ is so small, the curvature can be approximated to give

$$
k=\frac{\partial^{2} \bar{h}}{\partial \bar{x}^{2}}
$$

and thus the boundary condition (15) gives

$\frac{\partial \bar{P}}{\partial \bar{x}}=-\frac{\varepsilon}{C a} \frac{\partial^{3} \bar{h}}{\partial \bar{x}^{3}}$

substituting equation (28) into (27), we get

$\frac{\partial \bar{h}}{\partial t}=\frac{\in^{2} \bar{h}^{2}}{3}\left(\frac{\varepsilon}{C a} \frac{\partial^{3} \bar{h}}{\partial \bar{x}^{3}}-\frac{\beta}{C a}\right) \frac{\partial \bar{h}}{\partial \bar{x}}$

equation (29) is the governing equation of the thin liquid film.

\section{Similarity method:}

We concentrated on equation (29) since it represents the flow of a vertical film down a substrate. We have assumed through this work that the film thickness is constant far behind the front and this assumption is true since the fluid thins out there and so we apply the similarity method to see how this thinning process evolves in time.

Far behind the front $\left(\frac{\partial^{3} \bar{h}}{\partial \bar{x}^{3}}\right)$ is very small and thus we can neglect and so equation (29) reduces to give

$\frac{\partial \bar{h}}{\partial t}+\frac{\in^{2} \beta}{3 C a} \bar{h}^{2} \frac{\partial \bar{h}}{\partial \bar{x}}=0$

for self-similar solution, we assume that

$\bar{h}(\bar{x}, t)=h_{\circ}(t) \bar{H}(\eta)$

where $\eta$ is the self similar variable measures the distance to the front and

$\eta=\frac{\bar{x}}{\bar{x}_{f}(t)}, 0 \leq \eta \leq 1$

where $\bar{x}_{f}(t)$ is the front position as shown in figure (1), this method is to isolate the explicit time dependence and then the shape of the film will depend on the variable $\eta$ only. 
Now let

and

$$
\left.\begin{array}{l}
h_{\circ}(t)=A_{1} t^{\beta} \\
\bar{x}_{f}(t)=A_{2} t^{\delta}
\end{array}\right\}
$$

where $\beta$ and $\delta$ are the self similar exponents.

from (31) and (32), equation (30) gives

$\frac{\bar{H}}{h_{\circ}} \frac{\left(d h_{\circ} / d t\right) \bar{x}_{f}}{\left(d \bar{x}_{f} / d t\right)}-\eta \frac{d \bar{H}}{d \eta}+\frac{\beta \in^{2} \bar{H}^{2} h_{0}^{2}}{3 C a\left(d \bar{x}_{f} / d t\right)} \frac{d \bar{h}}{d \eta}=0$

Since the total volume of the fluid is constant, so the volume per unit width (volume flow rate) is also constant, thus

$\bar{u}=h_{\circ} \bar{x}_{f} \int_{0}^{1} \bar{H}(\eta) d \eta=$ constant

Which gives (since also $\int_{0}^{1} \bar{H}(\eta) d \eta=$ constant)

$\bar{x}_{f} \frac{d h_{\circ}}{d t}+h_{\circ} \frac{d \bar{x}_{f}}{d t}=0$

From (33) and (35), and since each of the terms in equation (34) is independent explicitly on $t$, we have

$\delta=-\beta=\frac{1}{3}$

Thus equation (34) gives

$\bar{H}+\eta \frac{d \bar{H}}{d \eta}-\frac{\beta A_{1}^{2} \in^{2}}{C a A_{2}} \bar{H}^{2} \frac{d \bar{H}}{d \eta}=0$

or

$d(\eta \bar{H})-\frac{\beta A_{1}^{2} \in^{2}}{\operatorname{Ca} A_{2}} \bar{H}^{2} d \bar{H}=0$

Integrating equation (36), we get

$\eta \bar{H}-\frac{\beta A_{1}^{2} \in^{2}}{3 C a A_{2}} \bar{H}^{3}=C$

where $C$ is a constant of integration since at $\eta=0$, we have $\bar{H}=0$, then from equation (37) we have $C=0$, and thus (37) gives

$\bar{H}(\eta)=\frac{1}{A_{1}}\left(\frac{3 C a A_{2} \eta}{\epsilon^{2} \beta}\right)^{\frac{1}{2}}$ 
Since $\bar{u}=h_{\circ} x_{f_{\circ}} \int_{0}^{1} \bar{H}(\eta) d \eta$, so (38) gives

$\bar{u}=A_{2}^{3 / 2} \frac{3 C a}{\beta \epsilon^{2}} \int_{0}^{1} \eta^{\frac{1}{2}} d \eta$

Integrating, we get

$A_{2}=\left(\frac{3 \beta}{4 C a} \in^{2} \bar{u}^{2}\right)^{\frac{1}{3}}$

From (33), we have

$\bar{x}_{f}=\left(\frac{3 \beta}{4 C a} \in^{2} t \bar{u}^{2}\right)^{\frac{1}{3}}$

Choose the constant $A_{1}=\frac{1}{3}$, equation (38), gives

$\bar{H}(\eta)=3 \frac{\left[\left(3 C a^{2 / 3} / 4 \bar{u}^{2}\right)^{\frac{1}{3}} \eta\right]^{-\frac{1}{3}}}{\left(\epsilon^{2} \beta\right)^{2 / 3}}$

Also from (32) and (40)

$\eta=\bar{x}\left(\frac{3 \in^{2} \beta \bar{u}^{2} t}{4 C a}\right)^{-\frac{1}{3}}$

from (41) and (4.2), we get after simplification,

$\bar{H}(\bar{x})=3 \sqrt{\frac{3 C a}{\epsilon^{2} \beta} \bar{x} t^{-\frac{1}{3}}}$

From the transformation (31), equation (42) gives

$\bar{h}(\bar{x}, t)=\sqrt{\frac{3 C a \bar{x}}{t \in^{2} \beta}}$

equation (44) represent the thickness of the film. Now the thickness at $x_{f}$ is then given by

$\bar{h}\left(\bar{x}_{f}, t\right)=\left(\frac{9 \bar{u} C a}{2 \epsilon^{2} \beta t}\right)^{\frac{1}{3}}$

equation (45) are used to determine the fluid thickness behind the capillary ridge.From (40), we have 
$t=\frac{4 x_{f}^{3} C a}{3 \epsilon^{2} \bar{u}^{2}}$

and so equation (45), gives

$\bar{h}\left(x_{f}\right)=\frac{3 \bar{u}}{2 x_{f}}$

Which gives the fluid thickness at $x_{f}$. and (4).

Some of the solution curves are presented in figures (2), (3)

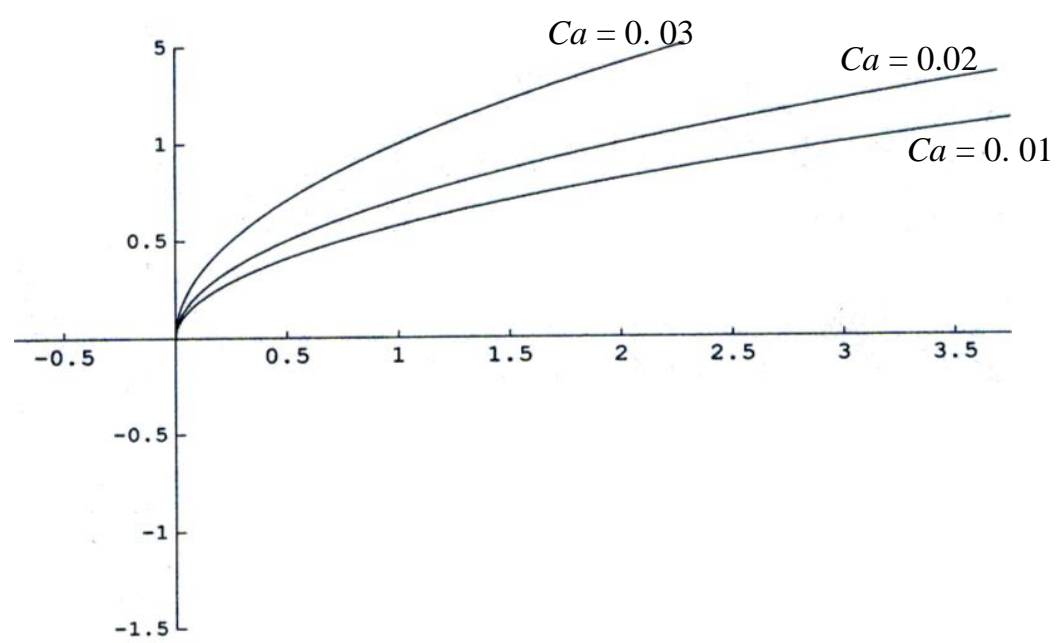

Figure (2): The thickness of the film for different capillary numbers $C a=0.01,0.02,0.03$ for fixed time $t=2$ and Bond number $=0.15$.

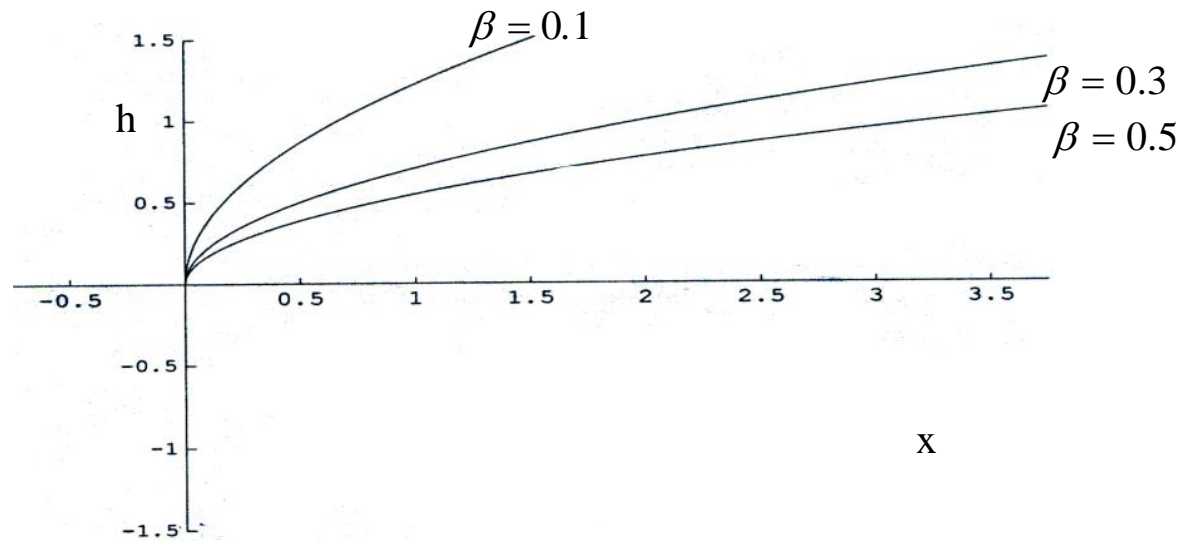

Figure (3): The thickness of the film for different Bond number $\beta=0.1,0.3,0.5$ and for fixed time $t=2$ and capillary number $C a=0.01$. 


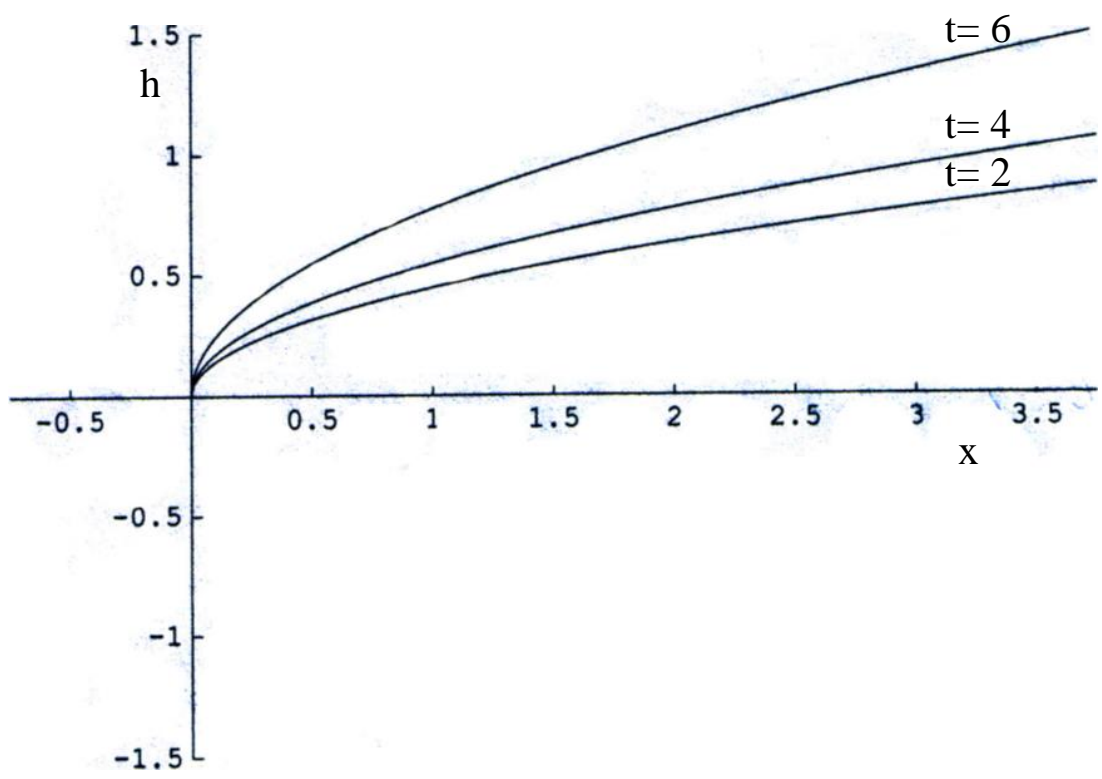

Figure (4): The thickness of the film for different time $t=2,4,6$ and for fixed Bond number $B=0.25$ and capillary number $C a=0.01$.

\section{Conclusion:}

We use the similarity method to solve differential equations for the drainage of a vertical liquid film and this method is very useful since the non-linear partial differential equations that governs such flow can be simplified to a single ordinary differential equations. The solution curves of the differential equation shows that the thickness of a liquid film increases as the time decrease and this usual. Furthermore, for different liquids such as glycerin and silicon the solution curves shows that the glycerin oil film is less than that of silicon oil film and the reason for this is the difference in the ratio of the bond and capillary numbers. 


\section{REFERENCES}

[1] Bertozzi, A.L. (1998). The mathematics of moving contact lines in thin liquid film, Amer. Math. Soc. Pp. 689-697, 45.

[2] Braun, R.J., Show S.A. and Pernisz, V.V. (1989). Gravitational drainage of a tangentially immobile thick film, Journal of Colloid and Interface Science, 219, 223-240.

[3] Diez, J., Kondic L. and Bertozzi, A.L. (2001). Global models for moving contact lines, Phys. Rev., pp. 011208, 63.

[4] Huppert H. (1982). Flow and instability of viscous current down a slope, Nature, pp. 427-429.

[5] Jerrett J.M., Debruyn, J.R. (1992). Finger instabilities of gravity driven contact line, Phys. Fluids, pp. 234-242, 4.

[6] Kondic L (2003). Instabilities in gravity driven flow of thin fluid films, Siam Review, vol. 45, No. 1, pp. 95-115.

[7] Kondic L. and Diez J. (1994). Contact line instabilities of thin fluid film flows: constant flux configuration, Phys. Fluids, pp. 224-234.

[8] Mysels K., Shinod K. and Frankel S. (1959). Soap film: studies of their thinning, Pregamon, New York 